\title{
Complications, Indication and Tolerance of Foam Sclerotherapy in Varicose Vein Management Done in A Tertiary Care Centre in South India
}

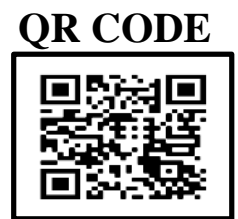

\section{PUNITHA THETRARAVU OLI', MAHITHA MC*2 (D)}

INTRODUCTION: Foam sclerotherapy is used for treating varicose veins in our institution for patients who are not willing for surgery debilitated patients, recurrent ulcers, cosmetic \& in SFV (Sapheno Femoral Vein) $<6 \mathrm{~mm}$ size.

AIM: The aim of this study was to find and assess the rate and pattern of post-sclerotherapy complications in our institution compared

A with international standards.

MATERIALS AND METHOD: The present study was retrospective in nature and case records of patients who underwent foam sclerotherapy [2009-2013, lower limb-Ultrasound (USG) guided and blind] in our hospital [10\% Sodiumtetradecylsulfate (STS), Tessari technique] were assessed to look for indication, tolerance to the procedure and immediate outcomes. A pre-tested and pre-validated questionnaire was used and physical examination were done to assess post-procedural complications. Statistical significance was $\mathrm{p}<0.05$. RESULTS: Among 112 patients, $62.5 \%$ were aged $>50$ years and $69 \%$ were males. It was observed that $22 \%$ had indication as recurrence; $43 \%$ had mild pain during procedure and $36.6 \%$ developed post-foam sclerotherapy complications. $47.6 \%$ of patients whose age were $<50$ years had CEAP(Clinical Etiological Anatomical Pathological) criteria $\mathrm{C}_{2}(\mathrm{p}=0.007) \cdot 48.8 \%$ with complications had multiple criteria( $\left.\mathrm{p}=0.012\right)$ and $57.7 \% \mathrm{C}_{2}$ had least complication $(\mathrm{p}=0.000$ ). Cosmetic indication showed least tolerance. Use of $10 \%$ STS was found to have lesser complication rate $(36 \%)$, compared to study which had $76 \%$ success with STS $3 \%$ and complication- $64 \%$.

CONCLUSION: Foam sclerotherapy is less invasive and its efficacy could be improved by performing under USG guidance, selection of sclerosant, appropriate candidate (age>50 years, multiple CEAP criteria, without pre-existing co-morbidities).

KEYWORDS: Varicose Vein, Sclerotherapy, Sclerosant

\section{INTRODUCTION}

Varicose veins are the most common vascular disease and it is due to incompetent valves, presenting with heaviness, aching and cramps of lower limbs. ${ }^{1}$ It is defined as dilated, usually tortuous, subcutaneous veins $\geq 3 \mathrm{~mm}$ in diameter measured in the upright position with demonstrable reflux. ${ }^{2}$

Its overall prevalence is $20 \%$ to $60 \%(25-30 \%$ among women and $15 \%$ in men). It has a genetic predisposition as when both parents are affected, there is a $90 \%$ chance for the offspring to get the disease and when one parent is affected, chances of inheritance in daughter is $60 \%$ and the chance of inheritance in son is $25 \% 3^{3}$ The prevalence of varicose vein depends on the following factors : gender, age(most commonly in the age group of $55-64$ years, and least in the age group of $18-24$ years), based on ethnicity, more prevalent among North America and Europe; least among South America and India. ${ }^{4}$ Other factors influencing prevalence are pregnancy, occupation and lifestyle (smokers, constipation, prolonged standing increases the risk). ${ }^{2}$ In a study among 100 teachers in Trivandrum, prevalence of varicose vein was $19 \%$. In a study among sweepers (men) it was $25.08 \%$ and $6.8 \%$ (South \& North India). ${ }^{5}$ About 20-25 million Americans have varicose veins. ${ }^{6}$
Treatment of varicose vein includes: conservative: compression stockings, intermittent pneumatic compression. Active:

1. Surgical: TSSA(Trendelenberg stripping and stab avulsion), SPJ (Sapheno Popliteal Junction)Ligation, ambulatory phlebectomy, cryosurgery.

2. Non-surgical : Sclerotherapy (reticular veins), endovenous thermal ablation.

In foam sclerotherapy which was introduced in 2001, lipid membranes(endothelial cells) are destroyed by sclerosant(detergent), by inducing sclerosis.

Tessari's Method: Here, sites are marked, and two syringes are connected by a three-way tap. 1:3 or 1:4 sclerosant: air is drawn into syringe and then oscillated. The foam is stable only for $\mathbf{2}$ minutes. The veins are emptied and foam is injected (1-2 ml, maximum 10-20 $\mathrm{ml}$ ) from superficial veins to Long Saphenous Vein or Short Saphenous Vein. Incidence of complications is directly proportional to the volume of sclerosant injected. Compression bandage is applied and left insitu for 7-10 days. ${ }^{2}$

Sclerosant approved by US FDA are 1. Sodium morrhuate and Sodium tetradecyl Sulfate. In UK, 
polidocanol is also used.7 The sclerosant used in our hospital is $10 \%$ Sodium tetradecyl sulphate.

The complication rate of foam sclerotherapy is $<1 \%$, which commonly includes phlebitis, pigmentation, headache, superficial skin necrosis, visual disturbances, chest tightness. ${ }^{1,2}$

Sclerotherapy, even if less invasive, is not widely used. In our hospital, sclerotherapy is carried out in patients who are not willing for surgery, debilitating patients, recurrent ulcers, cosmetic purposes and in patients with $<6 \mathrm{~mm}$ SFV size. The aim was to find out and assess the rate and pattern of post-sclerotherapy complications. The study also intended to analyse the complication rate of the procedure in US and UK and to find out whether the present methods are to be modified for better results.

\section{MATERIALS AND METHOD}

Ethical Consideration: This study was approved by Dr.SMCSI Medical College and Hospital ethical committee.

Method: The study group comprised of 112 patients who had undergone foam sclerotherapy (both ultrasound guided and blind sclerotherapy) during the years 2009-2013. Patients who were willing, had recurrent venous ulcers, debilitating, GSV size $<6 \mathrm{~mm}$, who had undergone foam sclerotherapy during 20092013 were included in the study. Those who had incomplete case records were excluded from the study. The study included dependent variables like superficial skin pigmentation at site of injection, pain at site of injection, skin necrosis, transient blindness and transient renal failure. Independent variables included name, age, sex, residence, morbidity.

Case records were studied for indications of sclerotherapy, details of the procedure, tolerance to the procedure and immediate outcomes. Data regarding post procedure followed upto 3 months and 6 months were obtained from case records. The data was entered in MS Excel and analysed using software (SPSS, version 22.0). Complication rate was computed as the total number of complications divided by the total subjects who had undergone foam sclerotherapy. Pattern of complications was assessed by taking the proportion of each complication.

\section{RESULTS}

Among 112 subjects, 77 were males and 35 were females. $37.5 \%$ belonged to the age group of $31-50$ years, $37.5 \%$ were aged $51-70$ years. $22 \%$ of the subjects underwent foam sclerotherapy due to recurrence either after surgery (mostly as thread veins) or previous foam sclerotherapy. $16 \%$ got it done for cosmetic benefits. Among the total subjects surveyed, only $81 \%$ had their data regarding tolerance to procedure being recorded. Among them, $43 \%$ experienced mild pain yet tolerable, while $20 \%$ had no pain and $5 \%$ had severe pain during the procedure. $97.32 \%$ of foam sclerotherapy were done under ultrasound guidance. Among the study subjects, $36.61 \%$ was found to have post-foam sclerotherapy complications, immediately after the procedure/within 3-6 months of procedure.

The most common complication was skin pigmentation (19\%) which lasted for more than 1 week after the procedure and for which patient had to revisit the general surgery OPD. Next common complication found was ulcer at the injection site, majority of whom had associated pain at the injection site. Next common complication was skin necrosis which took long time to settle. These complications needed hospitalisation. Other less common complications observed were Deep Vein Thrombosis (DVT) $(2 \%)$ and itching. Among these, itching associated with skin pigmentation/ulcer/necrosis had been excluded and included only those who presented with itching alone as the complaint which accounted for about $1 \%$. Recurrence after foam sclerotherapy was one of the main complications which led to repetition of foam sclerotherapy after first course. Renal failure was not found in any of the patients.

Among the subjects who had undergone foam sclerotherapy, $57 \%$ were in the age group $<5$ o years and $47.6 \%$ of them had CEAP (Clinical-EtiologicalAnatomical-Pathophysiological) criteria $\mathrm{C}_{2}$, next being more common than one criteria $\left(\mathrm{C}_{1}-\mathrm{C} 6\right)(\mathrm{p}=\mathrm{o.007})$. Those belonging to age $>50$ years, $47.9 \%$ had more than one CEAP criteria ( $\mathrm{p}=0.007$ ) and $37.5 \%$ had $\mathrm{C}_{2}$ criteria. $41.5 \%$ of subjects aged $<50$ years had ulcer as indication for foam sclerotherapy $(\mathrm{p}=0.015)$. 27.1\% of subjects aged $>5$ oyears presented with recurrence of varicose vein of lower limb as indication for foam sclerotherapy. Interestingly $25 \%$ of older age group presented in OPD for

cosmetic indication 


\begin{tabular}{|c|c|c|c|c|c|}
\hline $\begin{array}{c}\text { POST FOAM } \\
\text { SCLEROTHERAPY }\end{array}$ & \multicolumn{2}{|c|}{ PRESENT } & \multicolumn{2}{|c|}{ ABSENT } & $\begin{array}{l}\text { CHI SQUARE (p } \\
\text { VALUE) }\end{array}$ \\
\hline & $\mathrm{n}$ & $\%$ & $\mathrm{n}$ & $\%$ & \\
\hline \multicolumn{6}{|c|}{ 1. Age } \\
\hline$<50$ years & 23 & 56.1 & 41 & $57 \cdot 7$ & $0.029(\mathrm{p}=0.865)$ \\
\hline$>50$ years & 18 & 43.9 & 30 & 42.3 & \\
\hline \multicolumn{6}{|c|}{ 2. CEAP criteria } \\
\hline $\mathrm{Cl}_{1}$ & 1 & 2.4 & o & o & $2.025(\mathrm{p}=0.155)$ \\
\hline $\mathbf{C}_{2}$ & 7 & $17 \cdot 5$ & 41 & $57 \cdot 7$ & $16.886(\mathrm{p}=0.000)$ \\
\hline$C_{3}$ & 4 & 9.8 & 7 & $9 \cdot 9$ & $0.000(p=0.986)$ \\
\hline $\mathrm{C}_{4}$ & o & o & 1 & 1.4 & $0.917(p=0.338)$ \\
\hline$C_{5}$ & 1 & 2.4 & o & o & $2.025(\mathrm{p}=0.155)$ \\
\hline C6 & 7 & 17.1 & 4 & 5.6 & $3.677(\mathrm{p}=0.051)$ \\
\hline $\begin{array}{l}\text { Subjects having } \\
\text { more than one } \\
\text { CEAP criteria }\end{array}$ & 20 & 48.8 & 18 & 25.4 & $6.364(p=0.012)$ \\
\hline \multicolumn{6}{|c|}{ 3. Indication for foam sclerotherapy } \\
\hline A). Cosmetic & 8 & $19 \cdot 5$ & 10 & 14.1 & $0.568(\mathrm{p}=0.451)$ \\
\hline B). Skin changes & 13 & 31.7 & 4 & 5.6 & $13 \cdot 724(\mathrm{p}=0.000)$ \\
\hline C). Edema & 7 & 17.1 & 9 & 12.7 & $0.410(p=0.522)$ \\
\hline D). Ulcer & 17 & 41.5 & 8 & 11.3 & $13.668(\mathrm{p}=0.000)$ \\
\hline $\begin{array}{l}\text { E). Minor } \\
\text { abrasion }\end{array}$ & 1 & 2.4 & 11 & $15 \cdot 5$ & $5.644(\mathrm{p}=0.018)$ \\
\hline F). Recurrence & 16 & 39 & 8 & 11.3 & $11.893(\mathrm{p}=0.001)$ \\
\hline \multicolumn{6}{|c|}{ 4. Mode of foam sclerotherapy } \\
\hline A). USG guided & 39 & 95.1 & 70 & 98.6 & $1.145(\mathrm{p}=0.285)$ \\
\hline B). Blind & 2 & 4.9 & 1 & 1.4 & $1.145(\mathrm{p}=0.285)$ \\
\hline \multicolumn{6}{|c|}{ 5. Level of tolerance } \\
\hline a). No pain & 2 & $4 \cdot 9$ & 2 & 2.8 & $0.309(\mathrm{p}=0.578)$ \\
\hline b). Mild pain & 10 & $24 \cdot 4$ & 3 & 4.2 & $10.012(p=0.002)$ \\
\hline c). Severe pain & 5 & 7 & o & o & $4.692(p=0.030)$ \\
\hline
\end{tabular}

Table 1. Presence or absence of post foam sclerotherapy complication among the study subjects. (CEAP: Clinical-Etiological-Anatomical-Pathophysiological)

whereas it was only $9.4 \%$ among younger age group $(\mathrm{p}=0.026)$. Better tolerance to procedure was shown by those with age $<50$ years (14.1\%). 35.9\% with age $<50$ years and $37.5 \%$ with age $>50$ years had post foam sclerotherapy complications atleast within 6 months of procedure $(\mathrm{p}=0.827)$. Both the age groups had skin pigmentation as the most seen complication (18.8\%) with a $\mathrm{p}$ value $=0.978$.

Among subjects who had post foam sclerotherapy complications, $56.1 \%$ belonged to age $<50$ years $(\mathrm{p}=0.865) .48 .8 \%$ of those with complications belonged to multiple CEAP criteria $(\mathrm{p}=0.012)$ and those with $\mathrm{C}_{2}$ criteria were found to have least complications ( $\mathrm{p}=0.000$, table 1$) .41 .5 \%$ of subjects who had complications was found to have ulcer as their indication for foam sclerotherapy and the relation was found to be statistically significant ( $\mathrm{p}=0.000$, table 1 ). Next common indication was recurrent varicose vein $(39 \%)$ and skin changes $(31.7 \%)$, with a statistically significant relation to occurrence of complication ( $p$ value of 0.001 and $\mathrm{p}=0.000$ respectively). Those with 


\begin{tabular}{|c|c|c|c|c|c|}
\hline TOLERANCE TO & \multicolumn{2}{|c|}{ YES } & \multicolumn{2}{|c|}{ NO } & \multirow[t]{2}{*}{ CHI SQUARE (p VALUE) } \\
\hline & $\mathrm{n}$ & $\%$ & $\mathrm{n}$ & $\%$ & \\
\hline \multicolumn{6}{|c|}{ 1. Age } \\
\hline$<50$ years & 1 & 1.6 & 63 & 58.3 & \multirow[t]{2}{*}{$1.767(\mathrm{p}=0.184)$} \\
\hline$>50$ years & 3 & 75 & 45 & 41.7 & \\
\hline \multicolumn{6}{|c|}{ 2. CEAP criteria } \\
\hline $\begin{array}{l}\text { 2. CEAP multiple } \\
\text { criteria present }\end{array}$ & 3 & 75 & 1 & 25 & $2.928(\mathrm{p}=0.087)$ \\
\hline \multicolumn{6}{|c|}{ 3. Indication for foam sclerotherapy } \\
\hline a)cosmetic & o & o & 18 & 16.7 & $1.420(\mathrm{p}=0.232)$ \\
\hline b)skin changes & 1 & 25 & 16 & 14.8 & $0.271(p=0.603)$ \\
\hline c)edema & 2 & 50 & 14 & 13 & $3.014(p=0.083)$ \\
\hline d)ulcer & 2 & 50 & 23 & 21.3 & $1.530(\mathrm{p}=0.216)$ \\
\hline e)minor abrasion & o & o & 12 & 11.1 & $0.924(p=0.336)$ \\
\hline f)recurrence & o & o & 24 & 22.2 & $1.969(\mathrm{p}=0.161)$ \\
\hline \multicolumn{6}{|c|}{ 4. Complication present } \\
\hline $\begin{array}{c}\text { 4.complication } \\
\text { present }\end{array}$ & 2 & 50 & 39 & 36.1 & $0.309(\mathrm{p}=0.578)$ \\
\hline \multicolumn{6}{|c|}{ 5. Post foam sclerotherapy complication } \\
\hline A). Skin necrosis & o & o & 11 & 10.2 & $1.596(p=0.450)$ \\
\hline B). Skin pigmentation & 1 & 25 & 20 & 18.5 & $0.311(p=0.856)$ \\
\hline c). DVT & o & o & 2 & 1.9 & $0.514(p=0.773)$ \\
\hline D). Itching & 2 & 50 & 5 & 4.6 & $7.916(\mathrm{p}=0.019)$ \\
\hline E). Headache & o & o & 1 & .9 & $0.411(p=0.814)$ \\
\hline F). Ulcer & 1 & 25 & 12 & 11.1 & $0.613(p=0.736)$ \\
\hline G). Renal failure & o & o & o & o & o \\
\hline H). Recurrence & 1 & 25 & 7 & 87.5 & $1.301(\mathrm{p}=0.522)$ \\
\hline
\end{tabular}

Table 2. Tolerance of foam sclerotherapy among the study subjects (CEAP: Clinical-Etiological-Anatomical-Pathophysiological)

minor abrasion as the indication for foam sclerotherapy were found to have least (15.5\%) occurrence of complications ( $\mathrm{p}=\mathrm{o} .018)$ (Table 1 ).

It was observed that $60.5 \%$ of subjects having multiple CEAP criteria belonged to age $>5$ oyears. Among subjects who presented with multiple CEAP criteria, $47.4 \%$ presented with ulcer, $31.6 \%$ with edema as their indication for foam sclerotherapy. $17.6 \%$ of those with single CEAP criteria had cosmetic indication. 97.4\% of subjects with multiple CEAP criteria had undergone with USG guided foam sclerotherapy. Post foam sclerotherapy complications were present in $52.6 \%$ of subjects having multiple CEAP criteria- $\mathrm{p}$ value of 0.012 . Statistically significant relation was found among presence of multiple CEAP criteria and occurrence of all post-foam sclerotherapy complications were studied.

Majority $(75 \%)$ of subjects of older age group (>50years) and having multiple CEAP criteria were found to tolerate the procedure better than those of younger age group $(\mathrm{p}=0.184 \& \mathrm{p}=0.087$ respectively $)$. It has been found that none of those having cosmetic indication for foam sclerotherapy could tolerate the procedure $(\mathrm{p}=\mathbf{0 . 2 3 2}) .50 \%$ of subjects who could not tolerate the procedure were later found to have postfoam sclerotherapy complications $(\mathrm{p}=\mathrm{o} .578)$. Subjects with post-foam sclerotherapy complications like skin necrosis $(\mathrm{p}=\mathrm{o} .45 \mathrm{o})$, DVT $(\mathrm{p}=\mathrm{o} .773)$, headache $(\mathrm{p}=\mathrm{o} .814)$ have not been found to tolerate the procedure (table 2 ).

\section{DISCUSSION}

There are various treatment choices for lower limb varicose veins. Foam sclerotherapy causes complete endothelial damage and eventually fibrosis of the entire vein wall without recanalization and locates the points of irregular flow in the veins permanently obliterated by the sclerosant injection. ${ }^{4}$ In our institution $10 \%$ sodium tetradecyl sulphate is used, a detergent sclerosant agent. With this, the valves that are permanently damaged can regain their normal functions. The most important aspect of the treatment is to minimize the local venous hypertension by 
interrupting the points of leakage into the superficial network by the perforating veins. The migration of sclerosing solution into isolated segments of the injected superficial vein (digital compression) will effectively close the perforator complex of perforators. The incompetent perforators are located almost always near changes in the skin and in typical locations in the legs. ${ }^{8}$

Among the 145 subjects selected for the study during the year 2009-2013 in our hospital, according to inclusion criteria and availability of proper data records, 112 were actually included in the study, 77 were males and 35 were females. Of this, $94.64 \%$ belonged to age $<70$ years, $57 \%$ were aged $<50$ years, $19.64 \%$ aged $<30$ years.

$22 \%$ of the subjects underwent foam sclerotherapy due to recurrence either after surgery (mostly as thread veins) or previous foam sclerotherapy or due to ulceration. $16 \%$ got it done for cosmetic benefits, $15 \%$ due to skin changes, $14 \%$ edema and $11 \%$ minor abrasions, in contrast to an Italian study by Tessari L et al. wherein $70 \%$ of the sclerotherapy sessions were performed on recurrent varices, or collaterals and 30\% for reticular varices and telangiectases. ${ }^{9}$

In another study, common indications found were (a) pain in the affected limb - 38 patients (76\%); (b) eczema - 40 patients $(80 \%)$; (c) oedema - 15 patients (30\%); (d) ulceration - 15 patients (30\%); (e) lipodermatosclerosis - 10 cases (20\%); and (f) telangiectasia and atrophie blanche - 2 cases (4\%), which reveals a similar group of indications but lesser prevalence in present study. The disparity may be because the above mentioned research done by Subbarao.et.al was based on dermatological outcome of the treatment and thus skin changes were more included as the indications. ${ }^{10}$

A research carried out by Fun SK on the etiology and symptomatology of varicose veins of lower limbs showed that patients having pain were 59\%, pigmentation was seen in $58 \%$, edema in $53 \%$, eczema in $29 \%$, ulceration in $28 \%$, bleeding in $21 \%$ and lipodermatosclerosis in $20 \%$ which correlates well with the current study in case of prevalence of ulcer as indication(22\%). ${ }^{11}$

Among the total subjects studied, only $81 \%$ had their data regarding tolerance to procedure being recorded.
Among them, $43 \%$ experienced mild yet tolerable pain, which was found to be a factor that increases the patient compliance to procedure and makes them willing for repeated sittings.

97.32\% cases were done under ultrasound guidance which improved the efficacy of the procedure by effectively localising the incompetence.

Among the study subjects, $36.61 \%$ were found to have post-foam sclerotherapy complications, immediately after the procedure/within 3-6 months of procedure, which goes in hand with the findings in study conducted by Subbarao.et.al, in which $64 \%$ had postfoam sclerotherapy complications like bruising-38\%, pigmentation- $10 \%$, recurrence- $4 \%$,DVT-2 $\% .^{10}$ This study showed a higher incidence of complications possibly due to inclusion of thrombophlebitis, which is considered as a part of mode of action of sclerosant and not taken into account in the present study.

Post-sclerotherapy complications observed in the present study were $10 \%$ skin necrosis, $19 \%$ skin pigmentation, $2 \%$ DVT, $1 \%$ itching, ulcer $12 \%$, recurrence $7 \%$ \& no renal failure. Whereas the above study had not reported any case of skin necrosis. ${ }^{10}$ Mostly observed post procedural complication was skin pigmentation (19\%) which lasted for more than 1 week after the procedure and for which patient had to revisit the general surgery outpatient department(OPD) and had undergone either repeat foam sclerotherapy or other treatment. Next most common complication found was ulcer at the injection site, majority of which had associated pain at the injection site. Next common was skin necrosis which required longest time period for recovery among the complications. These were the complications which had required hospitalisation. Other less common complications observed were DVT \& itching. Among this, itching associated with skin pigmentation/ulcer/necrosis had been excluded. Recurrence after foam sclerotherapy was one of the main complications which led to repetition of foam sclerotherapy after first course, among which some even had to undergo surgical avulsion of the vein due to initial sclerotherapy failure. Another complication which had been looked for was renal failure which had not been reported in any of the patients may be due to plenty of oral fluids after the procedure. Other possible causes for the above mentioned complications were excluded to maximum by detailed history and 
thorough physical examination and radiographic studies before attributing to foam sclerotherapy.

Among the subjects who underwent foam sclerotherapy, those belonging to age $<50$ years presented with tortuous asymptomatic lower limb veins $\left(C_{2}\right)$ and also with more than one criteria $\left(C_{1}-C 6\right)$ $(\mathrm{p}=\mathrm{o.007})$, showing complicated presentation. Such patients with multiple CEAP criteria showed statistically significant relation with more incidences of post sclerotherapy complications also. Younger age group presented more with ulcer as indication for the procedure whereas for older group it was recurrence which was probably due to time lapse after first occurrence of varicose vein. Interestingly $25 \%$ of older age group presented in OPD for cosmetic indication whereas it was only $9.4 \%$ among younger age group $(\mathrm{p}=0.026)$, yet they tolerated the procedure well.

Among subjects who had post foam sclerotherapy complications, $56.1 \%$ belonged to age $<50$ years $(\mathrm{p}=0.865)$.

$41.5 \%$ of subjects who had complications were found to have ulcer as their indication for foam sclerotherapy and the relation was found to be statistically significant $(\mathrm{p}=0.000)$. Next common indication was recurrent varicose vein $(39 \%)$ and skin changes $(31.7 \%)$, with a statistically significant relation to occurrence of complication with a $\mathrm{p}$ value of 0.001 and $\mathrm{p}=0.000$ respectively. Those with minor abrasion as the indication for foam sclerotherapy were found to have least $(15.5 \%)$ occurrence of complications $(\mathrm{p}=0.018)$. Among subjects who presented with multiple CEAP criteria, $47.4 \%$ presented with ulcer ( $\mathrm{p}=0.000$ ), $31.6 \%$ with edema $(\mathrm{p}=0.000)$ and $26.3 \%$ with skin changes $(\mathrm{p}=0.019)$ as their indication for foam sclerotherapy.

$17.6 \%$ of those with single CEAP criteria had cosmetic indication $(\mathrm{p}=0.547)$ for foam sclerotherapy. $97.4 \%$ of subjects with multiple CEAP criteria underwent a USG guided foam sclerotherapy ( $\mathrm{p}=0.982)$.

$18.4 \%$ of subjects with multiple criteria had mild pain during the procedure. Post foam sclerotherapy complications were found to be present in $52.6 \%$ of subjects having multiple CEAP criteria and the relation was found to be statistically significant with a $p$ value of 0.012 .

Statistically significant relation were found among presence of multiple CEAP criteria and occurrence of all post-foam sclerotherapy complications studied for : skin necrosis-18.4\% ( $\mathrm{p}=0.022)$, skin pigmentation31.6\% ( $\mathrm{p}=0.021)$, DVT-2.6\% ( $\mathrm{p}=0.043)$, itching-10.5\% $(\mathrm{p}=0.039)$, headache-2.6\% $\quad(\mathrm{p}=\mathrm{o.021}), \quad$ ulcer$15.8 \%(\mathrm{p}=0.042)$, recurrence-10.5\%( $\mathrm{p}=0.043)$.

Majority $(75 \%)$ of subjects in older age group ( $>50$ years) \& having multiple CEAP criteria were found to tolerate the procedure better than those in younger age group ( $\mathrm{p}=0.184 \& \mathrm{p}=0.087$ respectively). It has been found that none of those having cosmetic indication for foam sclerotherapy could tolerate the procedure $(\mathrm{p}=0.232)$. Those with recurrence of varicose vein in lower limb as the indication were also not found to tolerate the procedure $(\mathrm{p}=\mathrm{o.161})$.

Among the $81 \%$ of procedure tolerance data available, $50 \%$ of subjects who could not tolerate the procedure were later found to have post-foam sclerotherapy complications $(\mathrm{p}=0.578)$. Subjects who did not tolerate the procedure presented with post-foam sclerotherapy complications like skin necrosis $(\mathrm{p}=0.450)$, DVT $(\mathrm{p}=0.773)$, headache $(\mathrm{p}=0.814)$.

In short, regarding tolerance to procedure, older individuals and those with multiple criteria were found to show better tolerance than those having cosmetic indication and recurrence of varicose vein. Better tolerance may be because multiple criteria varicose veins were all ultrasound guided procedures, reducing local reactions thereby. Also poor tolerance had ended up in post procedure complications also.

Even if there are associated post procedural complications, sclerotherapy is found to be a safer and more compliant procedure which could manage many varicose cases on an OPD basis, as proved in a study by Kanter and Thibault, who in 1996 reported a $76 \%$ success rate at 24 months in treating saphenofemoral junction and great saphenous vein incompetence with STS 3\% solution. ${ }^{12}$ In order to control reflux in the small saphenous vein, Padbury et al. found that ultrasound guided sclerotherapy was successful. ${ }^{13}$ Barrett et al. found that microfoam ultrasound guided sclerotherapy was effective in treating all sizes of varicose veins with high patient satisfaction and improvement in quality of life. $^{14}$

According to Cochrane Collaboration review of the medical literature "the evidence supports the current place of sclerotherapy in modern clinical practice, which is usually limited to treatment of recurrent varicose veins following surgery and thread veins". ${ }^{15} \mathrm{~A}$ 
second Cochrane Collaboration review comparing surgery to sclerotherapy found out that sclerotherapy was better than surgery in terms of treatment success, complication rate and cost at one year, but surgery was better after five years. ${ }^{16}$

The European Consensus Meeting on Foam Sclerotherapy in 2003 concluded that "Foam sclerotherapy allows a skilled practitioner to treat larger veins including saphenous trunks". ${ }^{17}$

When so much evidence is given on the clinical success of a procedure which had been evolving over a decade to the present state, more research could aid to overcome present complications too, making it a better resort to varicose vein management.

\section{CONCLUSION}

On the grounds of available knowledge on benefits of foam sclerotherapy, despite of the presence of postprocedure complications, it must be increasingly advocated to patients in indicated cases. Efficacy could be enhanced by performing under ultrasound guidance, selection of appropriate sclerosant, appropriate candidate. In the current study appropriate candidates were found to be older people with multiple CEAP criteria, without systemic illnesses who may predispose to complications studied herewith.

Acknowledgement: The authors would like to convey their sincere thanks to Dr. K. Raveendran, Professor and Head of Department, Dept. of General Surgery, Dr. SMCSI Medical College who had granted his valuable support and guidance for conducting this study and Dr. Suhana Shamsuddeen for her valuable support.

\section{REFERENCES}

1. Solomon AC, Maurya DK. Ultrasound guided foam sclerotherapy for varicose veins using two needle technique - A case series. Indian J Surg. 2010;72:249-51. https://doi.org/10.1007/s12262-010-0048-x

2. Bailey, Love. Short practice of surgery. (26th ed.) London: Hodder Arnold; 1991.

3. Callam MJ. Epidemiology of varicose veins. Br J Surg. 1994

Feb;81(2):167-73.

https://doi.org/10.1002/bjs.180o810204

4. Eklof B, Rutherford RB, Bergan JJ. Revision of the CEAP classification for chronic venous disorders: consensus statement. J Vasc Surg. 2004;50(6): 1248-52. https://doi.org/10.1016/j.jvs.2004.09.027
5. Malhotra SL. An epidemiological study of varicose veins in Indian railroad workers from the south and north India with special reference to causation and prevention of varicose veins. Int J Epid. 2011;1(2):177-83. https://doi.org/10.1093/ije/1.2.177

6. Tan VKM, Tan SG. Technique and early results of ultrasound-guided foam sclerotherapy of the long saphenous vein for treatment of varicose veins. Singapore Med J. 2009;50(3): 284-7.

7. Robert L. Wrothington-Kirsch. Injection Sclerotherapy. Semin Intervent Radiol. 2005;22(3):20917. https://doi.org/10.1055/s-2005-921954

8. Labas P, Ohradka B, Cambal M, Reis R, Fillo J. Long term results of compression sclerotherapy. Bratisl Lek Listy. 2003;104(2):78-81.

9. Tessari L, Cavezzi A, Frullini A. Preliminary experience with a new sclerosing foam in the treatment of varicose veins. Dermatol Surg. 2001 Jan;27(1):58-60.

https://doi.org/10.1111/j.1524-4725.2001.00192.x

10. Subbarao NT, Aradhya SS, Veerabhadrappa NH. Sclerotherapy in the management of varicose veins and its dermatological complications. Indian J Dermatol Venereol Leprol 2013; 79:383-8. https://doi.org/10.4103/0378-6323.110746

11. Fun SK. Varicose veins of the lower limbs: A study of aetiology and symptomatology. Hong Kong Pract. 1988;10:3319-26.

12. Kanter A, Thibault P. Saphenofemoral junction incompetence treated by ultrasound-guided sclerotherapy, Dermatol Surg. 1996. 22: 648-52.

https://doi.org/10.1111/j.1524-4725.1996.tboo612.x

13. Padbury A, Benveniste GL. Foam Echosclerotherapy of the small saphenous vein. Australian and New Zealand Journal of Phlebology. 2004;8:5-8.

14. Barrett JM, Allen B, Ockelford A, Goldman MP. Microfoam ultrasound-guided sclerotherapy treatment for varicose veins in a subgroup with diameters at the junction of 1omm or greater compared with a subgroup of less than $10 \mathrm{~mm}$. Dermatol Surg 2004; $30: 1386-1390$ https://doi.org/10.1111/j.1524-4725.2004.30430.x

15. Michaels JA, Campbell WB, Brazier JE. Randomised clinical trial, observational study and assessment of cost-effectiveness of the treatment of varicose veins (REACTIV trial). Health Technol Assess. 2006;10(13): 1196. https://doi.org/10.3310/hta10130

16. Tisi PV, Beverley C, Rees A. Injection sclerotherapy for varicose veins. Cochrane Database Syst Rev. 2006; (4):CDoo1732.

https://doi.org/10.1002/14651858.CDoo1732.pub2

17. Breu FX, Guggenbichler S. European Consensus Meeting on Foam Sclerotherapy, April, 4-6, 2003, Tegernsee, Germany. Dermatol Surg. 2004;30(5):709- 
17; discussion 717. https://doi.org/10.1111/j.15244725.2004.30209.x

\section{Cite this article as:}

Oli PT, Mahitha MC. Complications, Indication and Tolerance of Foam Sclerotherapy in

Varicose Vein Management Done in A Tertiary Care Centre in South India. Int Healthc Res

J. 2021;4(10):OR7-OR14. https://doi.org/10.26440/IHRJ/0410.01379

AUTHOR AFFILIATIONS: ( ${ }^{*}$ Corresponding Author)

1. MBBS, MS (Fellow in Vascular Surgery), Dr. Somervell Memorial CSI Medical College ,Karakonam, Trivandrum

2. MBBS, Dr. Somervell Memorial CSI Medical College, Karakonam, Trivandrum (ORCID ID: https://orcid.org/oooo-ooo2-6775-4136)

Source of support: Nil, Conflict of interest: None declared
Contact Corresponding Author at: mahimahimeenuz[at]gmail[dot]com 